\title{
Changing attitudes toward needle biopsies of breast cancer in Shanghai: experience and current status over the past 8 years
}

This article was published in the following Dove Press journal:

OncoTargets and Therapy

9 October 2015

Number of times this article has been viewed

\section{Shuang $\mathrm{HaO}^{1,2}$ \\ Zhe-Bin Liu' ${ }^{1,2}$ \\ Hong Ling 1,2 \\ Jia-Jian Chen ${ }^{1,2}$ \\ Ju-Ping Shen ${ }^{1,2}$ \\ Wen-Tao Yang ${ }^{2,3}$ \\ Zhi-Min Shao 1,2,4,5}

'Department of Breast Surgery, Fudan University Shanghai Cancer Center, ${ }^{2}$ Department of Oncology, Shanghai Medical College, Fudan University, ${ }^{3}$ Department of Pathology, Fudan University Shanghai Cancer Center, ${ }^{4}$ Institute of Biomedical Science, Fudan University, ${ }^{5}$ Key Laboratory of Breast Cancer in Shanghai, Department of Breast Surgery, Fudan University Shanghai Cancer Center, Shanghai, People's Republic of China
Correspondence: Zhi-Min Shao;

Zhe-Bin Liu

Department of Breast Surgery, Fudan

University Shanghai Cancer Center,

270 Dongan Road, Shanghai, 200032,

People's Republic of China

Tel +862164434556

Fax +8621 64434556

Email zhimingshao@yahoo.com;

lysalzb@hotmail.com
Abstract: Diagnostic patterns in breast cancer have greatly changed over the past few decades, and core needle biopsy (CNB) has become a reliable procedure for detecting breast cancer without invasive surgery. To estimate the changing diagnostic patterns of breast cancer in urban Shanghai, 11,947 women with breast lesions detected by preoperative needle biopsy between January 1995 and December 2012 were selected from the Shanghai Cancer Data base, which integrates information from approximately $50 \%$ of breast cancer patients in Shanghai. The CNB procedure uses an automated prone unit, biopsy gun, and 14-gauge needles under freehand or ultrasound guidance and was performed by experienced radiologists and surgeons specializing in needle biopsies. Diagnosis and classification for each patient were independently evaluated by pathologists. Over the indicated 8-year period, biopsy type consisted of 11,947 ultrasound-guided core needle biopsies (UCNBs), 2,015 ultrasound-guided vacuum-assisted biopsies (UVABs), and 654 stereotactic X-ray-guided vacuum-assisted biopsies (XVABs). For all the 11,947 women included in this study, image-guided needle biopsy was the initial diagnostic procedure. Approximately $81.0 \%$ of biopsied samples were histopathologically determined to be malignant lesions, $5.5 \%$ were determined to be high-risk lesions, and $13.5 \%$ were determined to be benign lesions. The number of patients choosing UCNB increased at the greatest rate, and UCNB has become a standard procedure for histodiagnosis because it is inexpensive, convenient, and accurate. The overall false-negative rate of CNB was $1.7 \%$, and the specific false-negative rates for UCNB, UVAB, and XVAB, were $1.7 \%, 0 \%$, and $0 \%$, respectively. This study suggests that the use of preoperative needle biopsy as the initial breast cancer diagnostic procedure is acceptable in urban Shanghai. Preoperative needle biopsy is now a standard procedure in the Shanghai Cancer Center because it may reduce the number of surgeries needed to treat breast cancer.

Keywords: breast carcinoma, core needle biopsy, ultrasound-guided core needle biopsies

\section{Introduction}

Despite the known benefits of needle biopsy for diagnosing suspicious breast lesions, variability in the use of this technique has been documented in practice. As a metropolis with rapid social and economic development over the past 3 decades, Shanghai has a breast cancer incidence that surpasses all other cancer registries in the People's Republic of China. ${ }^{1}$ Chinese patients have historically chosen open surgical biopsy (OSB) as the diagnostic method instead of needle biopsy due to the risk of triggering distant metastases. Over the last decade, however, breast cancer diagnosis in the People's Republic of China has greatly changed. Several disadvantages of traditional diagnostic procedures have been suggested, such as prolonged surgery time, drawbacks to neoadjuvant therapy, and surgery without a concrete pathological diagnosis. As a 
matter of course, it is important to make a rapid preoperative diagnosis to choose an appropriate treatment. Triple assessment, which consists of a physical examination, imaging modalities such as mammography and ultrasonography, and fine-needle aspiration, has been used to make a preoperative diagnosis. ${ }^{2}$ However, the development of core needle biopsy (CNB) for use with breast lesions has led to a gradual decline in the use of fine-needle aspiration. ${ }^{3}$ The aim of this study was to describe the trends and the present status of different diagnostic procedures in Shanghai and to analyze the various methods of obtaining core biopsies with respect to their diagnostic accuracy.

\section{Methods}

\section{Patient population}

To estimate the changing diagnostic patterns of breast cancer in urban Shanghai, all breast cancers (invasive and in situ) and all needle biopsies (benign or malignant) diagnosed between January 2005 and December 2012 were selected from the Shanghai Cancer Data Base, which integrates information from almost $50 \%$ of patients with breast cancer in Shanghai. This is a retrospective study of 11,947 consecutive needle biopsies performed by experienced radiologists and surgeons specialized in needle biopsies.

The study was conducted according to the principles expressed in the Declaration of Helsinki and approved by the institutional review board of Fudan University Shanghai Cancer Center. All the patients enrolled in this study signed the informed consent voluntarily.

\section{Biopsy method}

The surgeon and radiologist discussed the approach to each lesion, choosing the shortest route and skin entry site; they chose sites that could be comfortably included in future open surgery should cancer be diagnosed and avoided areas that could produce unsightly scars. After the radiographer had positioned the patient, the radiologist targeted the lesion and the surgeon performed the biopsy. The mode of percutaneous biopsy used was dependent upon the presenting lesion. The American College of Radiology guidelines for imageguided biopsy were followed. Ultrasound-guided core needle biopsy (UCNB) was preferentially used for all palpable and nonpalpable lesions that were visible by ultrasound. Approximately, five cores were taken from each lesion with a 14-gauge needle. Vacuum assistance was selectively used with UCNB based on tumor characteristics and was most often used for tumors that were less than $2 \mathrm{~cm}$ in size and considered benign. Stereotactic-guided biopsy was used for nonpalpable lesions not observed on ultrasonography, typically for microcalcifications or other imaging abnormalities observed by an initial mammography, but not identified by ultrasound. Vacuum assistance was used with stereotactic biopsies using a Suros (9-gauge) device, taking six to 12 cores per lesion. If the target lesion contained calcifications, a radiograph of the specimen was used to confirm the presence of calcifications in the cores. Analgesia for needle biopsies was accomplished with approximately 4-12 mL of buffered $1 \%$ lidocaine using a 26-gauge needle to anesthetize a cone of tissue around the lesion, as well as the needle track and associated skin. Skin punch biopsy was typically performed with a 4-mm instrument, and the wound was held together with DERMABOND $^{\circledR}$. The core tissue samples were immersed in $10 \%$ formalin solution and sent to the pathology laboratory for tissue processing.

\section{Statistical analysis}

Simple descriptive statistics, such as mean \pm standard deviation, median/range, and percentage were reported. The types of needle biopsies (core-needle or vacuum-assisted biopsy, X-ray or B-mode ultrasound-guided biopsy) and biopsy trends were analyzed. A secondary pathology review was performed for all needle biopsies, and patients whose cancer was diagnosed via needle biopsy were compared with patients whose cancer was diagnosed via surgical excision. When patients presented with more than one tumor, each cancer diagnosis was independently analyzed. Logistic regression was used to identify important predictors of biopsy type. By using multivariate logistic regression, we identified patient and tumor characteristics associated with the use of needle biopsy. We compared the histopathological findings of the needle biopsy specimens with those of surgical specimens or with each patient's long-term follow-up images. We also calculated the agreement, underestimation, sensitivity, and false-negative rates. The underestimation rate of CNB-diagnosed high-risk lesions was defined as the proportion of lesions diagnosed as high-risk by CNB that were upgraded to ductal carcinoma in situ (DCIS) or invasive cancer after surgical excision. The underestimation rate of CNB-diagnosed DCIS lesions was defined as the proportion of lesions diagnosed as DCIS by CNB, but upgraded to invasive cancer after surgical excision. Lesions which were not removed by subsequent surgical excision were excluded from the underestimation rate analysis. The false-negative rate was defined as the proportion of all breast cancers with a benign, high-risk, or inconclusive diagnosis on UCNB. 
Table I Ultrasound-guided core needle biopsies in the Shanghai Cancer Center

\begin{tabular}{|c|c|c|c|c|c|c|c|c|c|}
\hline Biopsies & 2005 & 2006 & 2007 & 2008 & 2009 & 2010 & 2011 & 2012 & $\overline{\text { Total }}$ \\
\hline Total, N & 167 & 606 & $\mathrm{I}, 00 \mathrm{I}$ & $\mathrm{I}, 245$ & 1,510 & $1,93 \mid$ & 2,375 & 3,112 & 11,947 \\
\hline Total (\%) & $(100)$ & $(100)$ & $(100)$ & $(100)$ & $(100)$ & $(100)$ & $(100)$ & $(100)$ & $(100)$ \\
\hline Malignant, $\mathrm{n}$ & 106 & 516 & 865 & 959 & $\mathrm{I}, 205$ & 1,510 & 1,923 & 2,589 & 9,673 \\
\hline Malignant (\%) & $(63.5)$ & $(85.1)$ & $(86.4)$ & $(77.0)$ & $(79.8)$ & $(78.2)$ & $(81.0)$ & $(83.2)$ & $(81.0)$ \\
\hline High-risk, n & 17 & 31 & 42 & 103 & 90 & 93 & 99 & 180 & 655 \\
\hline High-risk (\%) & $(10.2)$ & $(5.1)$ & $(4.2)$ & (8.3) & $(6.0)$ & $(4.8)$ & $(4.2)$ & $(5.8)$ & $(5.5)$ \\
\hline Benign, $n$ & 44 & 59 & 94 & 183 & 215 & 328 & 353 & 343 & 1619 \\
\hline Benign (\%) & $(26.3)$ & $(9.7)$ & $(9.4)$ & (I4.7) & $(14.2)$ & $(I 7.0)$ & $(14.8)$ & (II) & (13.5) \\
\hline
\end{tabular}

Notes: High-risk refers here to inadequate samples, lesions of uncertain malignant potential, and lesions where malignancy is suspected.

\section{Results}

\section{Constitution of preoperative diagnostic procedures in the Shanghai Cancer Center from 2005 to 2012}

Over the 8-year period from 2005 to 2012, the Shanghai Cancer Center registered 11,947 UCNBs, 2,015 ultrasoundguided vacuum-assisted biopsies (UVABs), and 654 stereotactic X-ray guided vacuum-assisted biopsies (XVABs; Tables 1 and 2). A total of $81 \%$ of lesions diagnosed by CNB were determined to be malignant $(n=9,673)$, whereas $5.5 \%$ were determined to be high-risk ( $\mathrm{n}=655)$, and $13.5 \%$ were determined to be benign ( $\mathrm{n}=1,619$; Table 1$)$. In these analyses, samples were determined to be high-risk when there was inadequate sample or a lesion of uncertain malignant potential as well as when malignancy was suspected. Image-guided needle biopsy was the initial procedure for approximately $80 \%(9,673$ of 11,947$)$ women who were considered to have malignant lesions. A diagnosis of cancer was made using microinvasive techniques for 106 of $167(63.5 \%)$ cancers in the year 2005 and for 2,589 of 3,112 (83.2\%) cancers in the year 2012, with an obvious rising trend.

Table 2 The pathologic results of UVAB and XVAB over the past 8 years

\begin{tabular}{|c|c|c|c|c|c|c|}
\hline \multirow[t]{2}{*}{ Year } & \multicolumn{3}{|c|}{ Ultrasound } & \multicolumn{3}{|l|}{ X-ray } \\
\hline & Total & Malignant & ADH & Total & Malignant & ADH \\
\hline 2004 & 68 & I & 0 & 50 & 7 & 6 \\
\hline 2005 & 83 & I & 1 & 38 & 7 & 5 \\
\hline 2006 & 127 & 0 & 0 & 88 & 22 & 1 \\
\hline 2007 & 152 & I & 0 & 83 & 14 & 3 \\
\hline 2008 & 161 & 0 & 4 & 115 & 18 & 3 \\
\hline 2009 & 279 & 4 & 0 & 79 & 5 & 0 \\
\hline 2010 & 440 & 3 & 2 & 110 & 16 & 7 \\
\hline 2011 & 705 & 25 & 7 & 91 & 13 & 2 \\
\hline 2012 & $\mathrm{I}, 027$ & 28 & II & 87 & 10 & 3 \\
\hline Total & 2,015 & 35 & 14 & 654 & 102 & 27 \\
\hline
\end{tabular}

Abbreviations: $A D H$, atypical ductal hyperplasia; UVAB, ultrasound-guided vacuum-assisted biopsy; XVAB, X-ray guided vacuum-assisted biopsy.
However, only $49(2.4 \%)$ and $129(19.7 \%)$ of lesions were diagnosed as malignant or atypical on UVAB and stereotactic $\mathrm{XVAB}$, respectively. The detailed pathological diagnoses for the malignant lesions and atypical ductal hyperplasia (ADH) identified by UVAB and stereotactic XVAB are presented in Table 2. When compared with year 2005, the number of UCNB, UVAB, and XVAB performed in 2012 increased by 18 -fold, 12-fold, and twofold, respectively. The lesions diagnosed by UVAB were predominantly benign, (1,966 of 2,015 [97.6\%] cases), whereas 35 cases diagnosed by UVAB were malignant; $80.3 \%$ of lesions diagnosed by $\mathrm{XVAB}$ were benign.

\section{Trends in the patients choosing CNB versus OSB}

Figure 1 shows the percentage of patients undergoing CNB or OSB. The diagnostic patterns greatly changed during the past 8 years, with increasing numbers of patients choosing preoperative needle biopsy instead of intraoperative frozen section analysis. For patients who were diagnosed and ultimately underwent surgery in our hospital, the number of preoperative needle biopsies greatly increased. The ratio of lesions diagnosed by preoperative biopsy to those diagnosed by intraoperative frozen section analysis tremendously increased from $0.14(103 / 731)$ to $4.6(2,148 / 468)$.

Figure 2 shows the composition of diagnostic patterns of the patients who chose OSB in 2005 and 2012. Significant changes occurred in the lesion pattern from 2005 to 2012 . In the year 2012, patients whose lesions were considered malignant were more likely to choose needle biopsy (388 of 731 , or $53.16 \%$ ) than surgical biopsy as their diagnostic procedure. Only patients who were not eligible for UCNB chose surgical biopsy as their diagnostic approach in 2012. Patients whose lesions were considered benign were also likely to choose surgical biopsy because benign lesions can be thoroughly treated using surgical biopsy. 


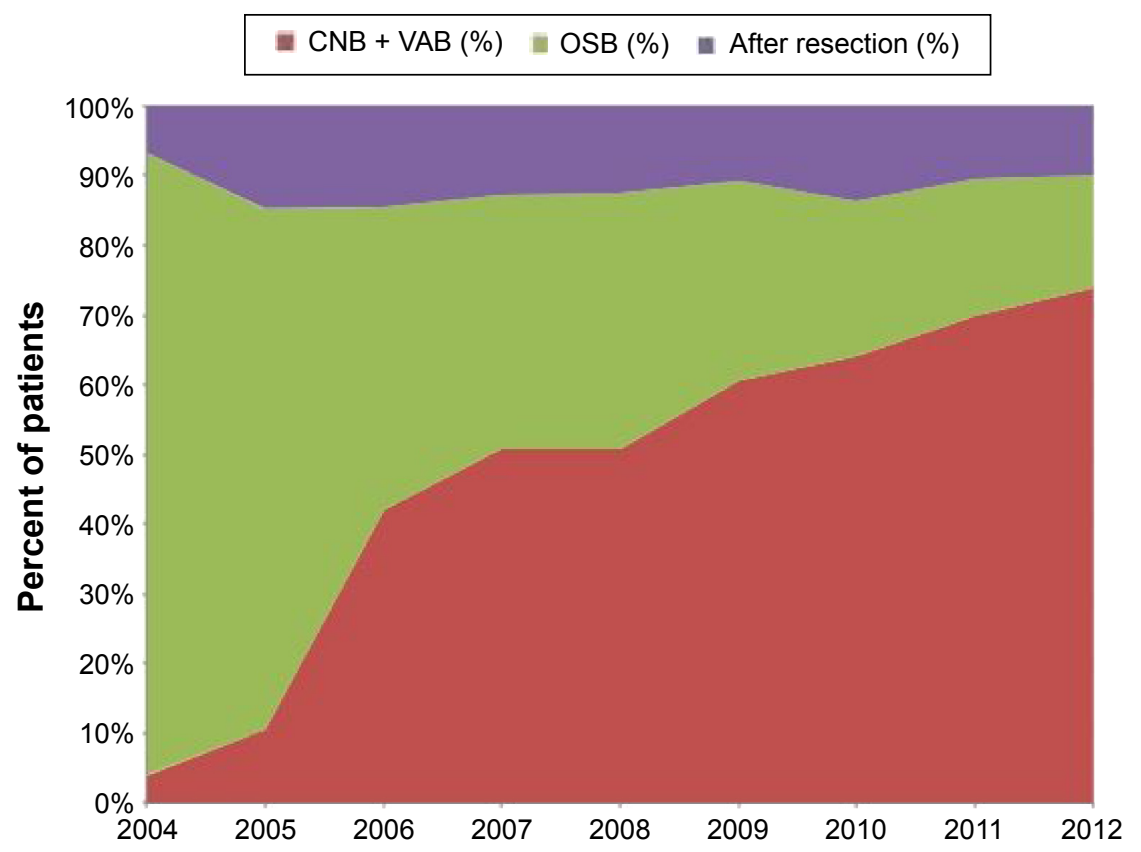

Figure I Trends in CNB and OSB procedures.

Notes: "After resection" refers to patients treated at our institution but diagnosed elsewhere; these were excluded.

Abbreviations: CNB, core needle biopsy; VAB, vacuum-assisted biopsy; OSB, open surgical biopsy.

\section{Logistic regression model estimating the likelihood of initial needle biopsy}

Table 3 shows the results of a multivariate logistic regression model estimating the likelihood of initial needle biopsy. Women who came from the provinces near Shanghai were more likely to receive a needle biopsy than women living in Shanghai (odds ratio, 1.12; $95 \%$ confidence interval [CI],
1.01-1.25), after controlling for other variables. Women with tumors of lower stage, smaller size, or lower grade were less likely to receive initial needle biopsy. After controlling for patient and tumor characteristics, the choice of needle biopsy was associated with year of diagnosis, which remained a significant predictor of initial needle biopsy on logistic regression analysis.
A

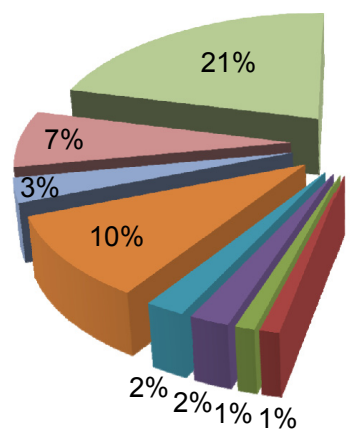

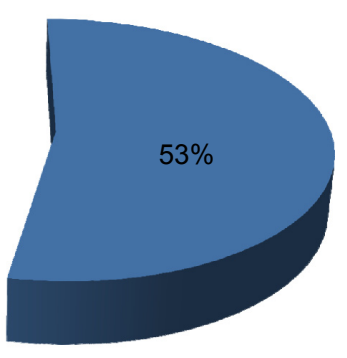

B

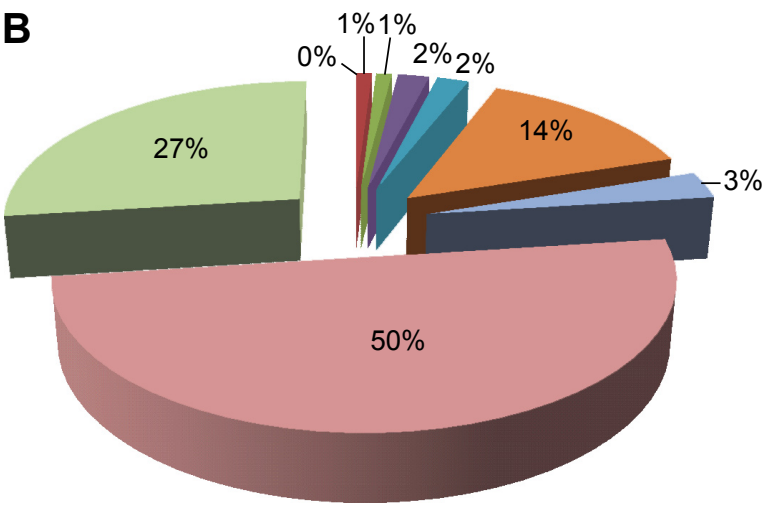

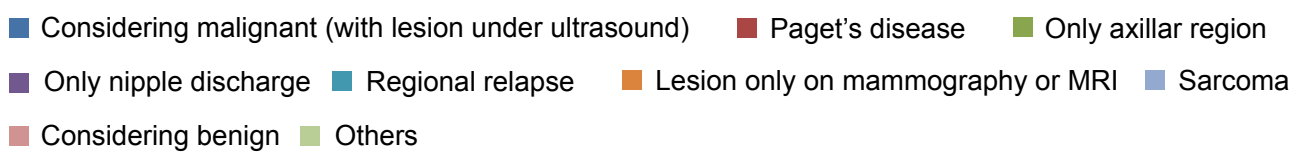

Figure 2 Distribution of the clinical characteristics of patients who chose OSB.

Notes: (A) Patients choosing open surgical biopsy in 2005. (B) Patients choosing open surgical biopsy in 2012.

Abbreviations: OSB, open surgical biopsy; MRI, magnetic resonance imaging. 
Table 3 Patient, tumor, and system characteristics associated with needle biopsy as the initial diagnostic technique: the results of a multivariate logistic regression model

\begin{tabular}{|c|c|c|c|}
\hline \multirow{2}{*}{$\begin{array}{l}\text { Patient } \\
\text { characteristics }\end{array}$} & \multirow{2}{*}{$\begin{array}{l}\text { Odds } \\
\text { ratio }\end{array}$} & \multicolumn{2}{|l|}{$95 \% \mathrm{Cl}$} \\
\hline & & Lower & Upper \\
\hline Age at diagnosis & 0.93 & 0.87 & 1.02 \\
\hline \multicolumn{4}{|c|}{ Tumor characteristics } \\
\hline \multicolumn{4}{|l|}{ Stage } \\
\hline Stage 0 & 0.76 & 0.6 & 0.93 \\
\hline Stage I & 0.81 & 0.73 & 0.91 \\
\hline Stage 2 & 1.00 & - & - \\
\hline \multicolumn{4}{|l|}{ Tumor size } \\
\hline$<2 \mathrm{~cm}$ & 0.92 & 0.86 & 0.98 \\
\hline $2-5 \mathrm{~cm}$ & 0.90 & 0.76 & 1.06 \\
\hline Missing tumor size & 0.92 & 0.82 & 1.04 \\
\hline$>5 \mathrm{~cm}$ & 1.00 & - & - \\
\hline \multicolumn{4}{|l|}{ Grade } \\
\hline Grade I & 0.84 & 0.79 & 0.91 \\
\hline Grade 2 & 0.92 & 0.86 & 0.97 \\
\hline Grade 3 & 1.00 & - & - \\
\hline \multicolumn{4}{|c|}{ System characteristics } \\
\hline \multicolumn{4}{|l|}{ Birth city } \\
\hline Near province & 1.12 & 1.01 & 1.25 \\
\hline Other & 0.92 & 0.82 & 1.04 \\
\hline Shanghai & 1.00 & - & - \\
\hline \multicolumn{4}{|l|}{ Year of diagnosis } \\
\hline $2005-2006$ & 0.74 & 0.68 & 0.80 \\
\hline 2007-2009 & 0.89 & 0.85 & 0.94 \\
\hline $2010-2012$ & 1.00 & - & - \\
\hline
\end{tabular}

Abbreviation: $\mathrm{Cl}$, confidence interval.

\section{Agreement between initial preoperative (needle biopsy) diagnosis and final pathological diagnosis}

Based on our results, we calculated the accuracy, underestimation rate, and false-negative rate of CNB (Table 4). The total accuracy rate for the past 8 years was $92.4 \%$, ranging from $89.8 \%$ in 2005 to $93.0 \%$ in 2012 with a gradual rising trend. The total underestimation rate of CNB-diagnosed lesions was $5.9 \%$ (709 of 11,947). These underestimation rates were calculated only for lesions that were subsequently excised surgically. The overall false-negative rate was $1.7 \%$, with specific false-negative rates for UCNB, UVAB, and XVAB of $1.7 \%, 0 \%$, and $0 \%$, respectively. The kappa measure of agreement between the UCNB results and surgical excision findings or follow-up results was $0.924(P<0.001)$. In the year 2012, for example, in 3,112 patients who were diagnosed by UCNB, the false-negative rate was $1.2 \%$ (36 of $3,112)$, and the sensitivity was $98.2 \%(3,076$ of 3,112$)$.

We defined an ADH underestimate as the percutaneous diagnosis of $\mathrm{ADH}$ in a lesion shown to have DCIS/DCIS microinvasion (DCIS-mi)/invasive ductal carcinoma on surgical histology, a DCIS underestimate as the percutaneous diagnosis of DCIS in a lesion shown to have invasive cancer on surgical histology, and a phyllodes tumor (PLT) underestimate as the percutaneous diagnosis of papillary lesion (PL) in a lesion shown to have DCIS/invasive ductal carcinoma/ intracystic papillary carcinomas on surgical histology. The overall accuracy rate of diagnostic $\mathrm{CNB}$ was $92.4 \%$, and 709 patients $(5.9 \%)$ had lesions that were underestimated, including 162 (22.8\%) ADH, 435 (61.4\%) DCIS, 91 (12.7\%) PL, and 21 (2.9\%) PLTs (Table 5).

\section{Discussion}

CNB is a reliable procedure for detecting breast cancer without invasive surgery. Open biopsy procedures are not required in patients with histologically benign percutaneous biopsy results, if the imaging and pathological findings are concordant. However, patients with $\mathrm{ADH}$, atypical lobular hyperplasia, or lobular carcinoma in situ found on percutaneous biopsy may have DCIS or invasive cancer at the same site and should generally undergo surgical excision.

The diagnostic accuracy of CNB is reported to be higher for nonpalpable lesions, ${ }^{4,5}$ and this procedure draws more attention from surgeons because they are impressed by the accuracy of CNB. CNB has been available in the People's

Table 4 The accuracy rate, underestimation rate, and false-negative rate of CNB

\begin{tabular}{lllll}
\hline Year & Accuracy rate (\%) & Underestimation rate (\%) & False-negative rate (\%) & Total (\%) \\
\hline 2005 & $150(89.8)$ & $13(7.8)$ & $4(2.4)$ & $167(100)$ \\
2006 & $562(92.7)$ & $32(5.3)$ & $12(2.0)$ & $606(100)$ \\
2007 & $917(91.6)$ & $56(5.6)$ & $28(2.8)$ & $1,001(100)$ \\
2008 & $1,154(92.7)$ & $68(5.5)$ & $23(1.8)$ & $1,245(100)$ \\
2009 & $1,380(91.4)$ & $95(6.3)$ & $35(2.3)$ & $1,510(100)$ \\
2010 & $1,773(91.8)$ & $124(6.4)$ & $34(1.8)$ & $1,931(100)$ \\
2011 & $2,204(92.8)$ & $140(5.9)$ & $31(1.3)$ & $2,375(100)$ \\
2012 & $2,895(93.0)$ & $181(5.8)$ & $36(1.2)$ & $3,112(100)$ \\
Total & $11,035(92.4)$ & $709(5.9)$ & $203(1.7)$ & $1,947(100)$ \\
\hline
\end{tabular}

Notes: The accurate rate was defined as the proportion of lesions correctly identified as benign, malignant, or high-risk by CNB; underestimation rate refers to lesions of uncertain malignant potential (upstaged to DCIS or invasive cancer), lesions of uncertain malignant potential excluding ADH or atypical lobular hyperplasia (upstaged to DCIS or invasive cancer), ADH (upstaged to DCIS or invasive cancer), and DCIS (upstaged to invasive cancer) that were underestimated.

Abbreviations: CNB, core needle biopsy; DCIS, ductal carcinoma in situ; ADH, atypical ductal hyperplasia. 
Table 5 Analysis of CNB underestimation

\begin{tabular}{lllll}
\hline $\begin{array}{l}\text { CNB } \\
\text { diagnosis }\end{array}$ & $\begin{array}{l}\text { Total } \\
\text { cases* }\end{array}$ & $\begin{array}{l}\text { Final } \\
\text { diagnosis }\end{array}$ & Cases** & $\begin{array}{l}\text { Percentage } \\
\text { of total cases }\end{array}$ \\
\hline ADH & 162 & DCIS & 51 & 32.6 \\
& & DCIS-mi & 56 & 35.8 \\
& & IDC $<$ I cm & 25 & 14.7 \\
& & IDC & 30 & 16.9 \\
DCIS & 435 & DCIS-mi & 202 & 49.2 \\
& & Mainly DCIS & 142 & 31.5 \\
& & IDC & 91 & 19.3 \\
PL & 91 & DCIS & 25 & 25.1 \\
& & IDC & 25 & 26.9 \\
& & IPC & 40 & 48 \\
Other & 21 & PLT, etc & 21 & 100 \\
Total & 709 & & 709 & 100 \\
\hline
\end{tabular}

Notes: *Total number of cases according to CNB diagnosis. **Number of cases according to final diagnosis.

Abbreviations: $\mathrm{CNB}$, core needle biopsy; $\mathrm{ADH}$, atypical ductal hyperplasia; $\mathrm{DCIS}$, ductal carcinoma in situ; DCIS-mi, DCIS microinvasion; IDC, invasive ductal carcinoma; IPC, intracystic papillary carcinomas; PLT, phyllodes tumor; PL, papillary lesion.

Republic of China for breast biopsies since the 1990s. For palpable masses, CNB is the first-line test, whereas imageguided CNB is used for the diagnosis of image-detected breast abnormalities. Freehand or UCNB is less invasive, more cost-effective, and less time-consuming than surgical biopsy and yields comparable results. ${ }^{6}$ There are relatively few patients for whom excisional biopsy should be the initial diagnostic procedure. For patients with a diagnosis of breast cancer, the goal is to make the diagnosis with a needle and to go to the operating room for treatment only once. A definitive diagnosis of breast cancer made using a minimally invasive needle biopsy permits optimal preoperative workup, patient counseling, and surgical planning.

Women commonly present in clinics for the evaluation of palpable breast masses in urban People's Republic of China. Compared with Western people, Chinese people lack awareness of breast self-examinations and health surveys, mostly because these issues receive less investment and less attention from the government. In the last decade, awareness of breast cancer has increased and diagnostic patterns have greatly changed in the People's Republic of China. CNB of the breast was introduced and combined with stereotactic (mammographic) or ultrasound guidance, the results were encouraging. ${ }^{7}$ Over the last 8 years, we have performed a large number of core biopsies on breast lesions under ultrasound guidance. Of these, $81.0 \%$ of the samples were histopathologically determined to be malignant lesions, $5.5 \%$ were determined to be high-risk lesions, and $13.5 \%$ were determined to be benign lesions. In the year 2012, 2,589 of $3,112(83.2 \%)$ cancers were determined to be malignant; this represents an obvious rising trend since 2005. Image-guided
CNB is very accurate and was an acceptable diagnostic procedure prior to OSB. ${ }^{8-12}$

The results of the present study confirmed that UCNB is an accurate and reliable method for the diagnosis of breast lesions. The sensitivity of CNB was reported to range from $94 \%$ to $99 \%$, and its specificity was reported to range from $99 \%$ to $100 \% \cdot{ }^{13}$ In our study, we reviewed 11,947 cases of $\mathrm{CNB}$ of which most were performed using 14-gauge needles, and approximately four cores were taken per lesion. Our data identified the presence of breast lesions in women undergoing CNB for the past 8 years with an overall accuracy of approximately $92.4 \%$, ranging from $89.8 \%$ in 2005 to $93.0 \%$ in 2012 with a gradual rising trend. The total underestimation rate of CNB-diagnosed lesions was $5.9 \%$ (709 of 11,947). It has been reported that needles larger than 16-gauge provide the most accurate diagnosis (in both sensitivity and specificity). ${ }^{14}$ These reports also stress the importance of the number of cores taken during each biopsy; the accuracy and sensitivity for detecting nonpalpable breast lesions increase when six or more tissue samples are taken. ${ }^{15,16}$

Over the past 10 years, minimally invasive breast biopsy systems have greatly developed. ${ }^{17-22}$ As a minimally invasive detection method, the vacuum-assisted system provides a new choice for the excision of benign breast lesions and has been successfully applied in the excision of benign breast lesions in previous studies. This method is commonly used in the People's Republic of China to completely remove lesions that are considered benign, because this procedure is too expensive and not covered by insurance.

A special phenomenon can be found in our data: women who came from the provinces surrounding Shanghai were more likely to receive a needle biopsy than women living in Shanghai, after controlling for other variables. In general, Chinese people approach medicine very conservatively; they preferred traditional diagnostic methods such as OSB and needle biopsy because of the risk of triggering distant metastasis and leaving residual lesions. Patients who traveled to Shanghai from other provinces for further treatment were willing to choose newer and more effective medical treatments; patients have a very strong sense of trust for doctors. The lack of medical coverage for $\mathrm{CNB}$ may be another reason for the increased rate of $\mathrm{CNB}$ among patients living outside Shanghai.

There are certain limitations of our study. For instance, as a hospital-based study, we described the trends and the present status of different diagnostic procedures in Shanghai and determined the core biopsy diagnostic accuracy. Wellreported retrospective chart reviews, retrospective data-base analyses, or prospective diagnostic accuracy studies are 
needed to address unanswered questions about which factors affect the accuracy and reliability of breast CNB. In the future, it is anticipated that the results of cost-effectiveness studies will provide opportunities to optimize the criteria for selection of appropriate procedures on an individual basis, considering costs, and should influence the decision about the choice of the optimal sampling procedure. ${ }^{23}$ In addition, further molecular biology analysis will be helpful to identify high-risk breast cancer patients who may benefit from the currently available breast biopsy methods.

\section{Conclusion}

The use of preoperative needle biopsies for initial breast cancer diagnosis is acceptable in urban Shanghai and is now a standard procedure at the Shang Cancer Center. We mostly use UCNB to diagnose malignant tumors. UCNB is more common among those with larger tumors, and its prevalence has significantly increased over time. UCNB is an accurate diagnostic alternative to surgical biopsy in patients with breast lesions detected via ultrasound, although the high underestimation rates for DCIS and high-risk lesions are still a concern. Providers should consider needle biopsy when clinically feasible as the initial breast diagnostic procedure, because it may reduce the number of surgeries needed to treat breast cancer.

\section{Disclosure}

The authors report no conflicts of interest in this work.

\section{References}

1. Fan L, Zheng Y, Yu KD, et al. Breast cancer in a transitional society over 18 years: trends and present status in Shanghai, China. Breast Cancer Res Treat. 2009;117(2):409-416.

2. Kurita T, Tsuchiya S, Watarai Y, et al. Roles of fine-needle aspiration and core needle biopsy in the diagnosis of breast cancer. Breast Cancer. 2012;19(1):23-29.

3. Tabbara SO, Frost AR, Stoler MH, Sneige N, Sidawy MK. Changing trends in breast fine-needle aspiration: results of the Papanicolaou Society of Cytopathology Survey. Diagn Cytopathol. 2000;22(2):126-130.

4. Wei X, Li Y, Zhang S, Zhu Y, Fan Y. Experience in large-core needle biopsy in the diagnosis of 1431 breast lesions. Med Oncol. 2011;28(2): 429-433.

5. Usami S, Moriya T, Kasajima A, et al. Pathological aspects of core needle biopsy for non-palpable breast lesions. Breast Cancer. 2005;12(4): $272-278$.

OncoTargets and Therapy

\section{Publish your work in this journal}

OncoTargets and Therapy is an international, peer-reviewed, open access journal focusing on the pathological basis of all cancers, potential targets for therapy and treatment protocols employed to improve the management of cancer patients. The journal also focuses on the impact of management programs and new therapeutic agents and protocols on
6. Schueller G, Schueller-Weidekamm C, Helbich TH. Accuracy of ultrasound-guided, large-core needle breast biopsy. Eur Radiol. 2008; 18(9):1761-1773.

7. Bolmgren J, Jacobson B, Nordenstrom B. Stereotaxic instrument for needle biopsy of the mamma. AJR Am J Roentgenol. 1977;129(1): $121-125$.

8. Wong TT, Cheung PS, Ma MK, Lo GG. Experience of stereotactic breast biopsy using the vacuum-assisted core needle biopsy device and the advanced breast biopsy instrumentation system in Hong Kong women. Asian J Surg. 2005;28(1):18-23.

9. Parker SH, Lovin JD, Jobe WE, Burke BJ, Hopper KD, Yakes WF. Nonpalpable breast lesions: stereotactic automated large-core biopsies. Radiology. 1991;180(2):403-407.

10. Elvecrog EL, Lechner MC, Nelson MT. Nonpalpable breast lesions: correlation of stereotaxic large-core needle biopsy and surgical biopsy results. Radiology. 1993;188(2):453-455.

11. Dronkers DJ. Stereotaxic core biopsy of breast lesions. Radiology. 1992;183(3):631-634.

12. Parker SH, Burbank F, Jackman RJ, et al. Percutaneous large-core breast biopsy: a multi-institutional study. Radiology. 1994;193(2):359-364.

13. Pisano ED, Fajardo LL, Caudry DJ, et al. Fine-needle aspiration biopsy of nonpalpable breast lesions in a multicenter clinical trial: results from the radiologic diagnostic oncology group V. Radiology. 2001;219(3): 785-792.

14. Nath ME, Robinson TM, Tobon H, Chough DM, Sumkin JH. Automated large-core needle biopsy of surgically removed breast lesions: comparison of samples obtained with 14-, 16-, and 18-gauge needles. Radiology. 1995;197(3):739-742.

15. Brenner RJ, Fajardo L, Fisher PR, et al. Percutaneous core biopsy of the breast: effect of operator experience and number of samples on diagnostic accuracy. AJR Am J Roentgenol. 1996;166(2):341-346.

16. Rich PM, Michell MJ, Humphreys S, Howes GP, Nunnerley HB. Stereotactic 14G core biopsy of non-palpable breast cancer: what is the relationship between the number of core samples taken and the sensitivity for detection of malignancy? Clin Radiol. 1999;54(6):384-389.

17. Fine RE, Israel PZ, Walker LC, et al. A prospective study of the removal rate of imaged breast lesions by an 11-gauge vacuum-assisted biopsy probe system. Am J Surg. 2001;182(4):335-340.

18. Fine RE, Boyd BA, Whitworth PW, Kim JA, Harness JK, Burak WE. Percutaneous removal of benign breast masses using a vacuumassisted hand-held device with ultrasound guidance. Am J Surg. 2002; 184(4):332-336.

19. Johnson AT, Henry-Tillman RS, Smith LF, et al. Percutaneous excisional breast biopsy. Am J Surg. 2002;184(6):550-554; discussion 554.

20. Chen SC, Yang HR, Hwang TL, Chen MF, Cheung YC, Hsueh S. Intraoperative ultrasonographically guided excisional biopsy or vacuumassisted core needle biopsy for nonpalpable breast lesions. Ann Surg. 2003;238(5):738-742.

21. March DE, Coughlin BF, Barham RB, et al. Breast masses: removal of all US evidence during biopsy by using a handheld vacuum-assisted device - initial experience. Radiology. 2003;227(2):549-555.

22. Wang ZL, Liu G, Huang Y, Wan WB, Li JL. Percutaneous excisional biopsy of clinically benign breast lesions with vacuum-assisted system: comparison of three devices. Eur J Radiol. 2012;81(4):725-730.

23. Dequanter D, Shahla M, Paulus P, Lothaire P. Long term results of sentinel lymph node biopsy in early oral squamous cell carcinoma. Onco Targets Ther. 2013;6:799-802.

\section{Dovepress}

patient perspectives such as quality of life, adherence and satisfaction. The manuscript management system is completely online and includes a very quick and fair peer-review system, which is all easy to use. Visit http://www.dovepress.com/testimonials.php to read real quotes from published authors. 